\title{
COMPLETE SOLUTIONS OF A COUPLED SYSTEM \\ OF PARTIAL DIFFERENTIAL EQUATIONS ARISING IN THERMOELASTICITY*
}

BY

\section{S. CHANDRASEKHARAIAH}

Bangalore University, Bangalore-560001, India

\begin{abstract}
Three general, complete solutions of a coupled hyperbolic or hyperbolicparabolic system of two second-order linear partial differential equations are presented. The system includes among its particular cases the governing field equations of the conventional as well as generalized thermoelasticity theories. The solutions obtained are analogous to the Lamé, Papkovitch, and Galerkin solutions in classical elasticity. The interrelationships among the solutions are also exhibited. Some solutions obtained in earlier works are deduced as special cases of the unified solutions obtained here.
\end{abstract}

1. Introduction. Suppose $\mathscr{D}$ is a regular region in the Euclidean three-space and $\mathscr{T}$ is a time interval. Consider the following system of partial differential equations defined over $\mathscr{D} \times \mathscr{T}:$

$$
\begin{gathered}
\left(c^{2} \nabla^{2}-\frac{\partial^{2}}{\partial t^{2}}\right) \mathbf{u}+\left(1-c^{2}\right) \nabla \operatorname{div} \mathbf{u}-\left(1+\alpha \frac{\partial}{\partial t}\right) \nabla \theta+\mathbf{F}=\mathbf{0}, \\
\left(\nabla^{2}-\frac{\partial}{\partial t}-\beta \frac{\partial^{2}}{\partial t^{2}}\right) \theta-\left(1+\gamma \frac{\partial}{\partial t}\right)\left[\varepsilon \frac{\partial}{\partial t}(\operatorname{div} \mathbf{u})-h\right]=0 .
\end{gathered}
$$

Here $c, \alpha, \beta, \gamma$, and $\varepsilon$ are real constants such that

$$
0<c<1, \alpha \geqslant 0, \beta \geqslant 0, \gamma \geqslant 0, \varepsilon \geqslant 0
$$

and $\mathbf{F}$ and $h$ are known functions (of the independent variables $\mathbf{x} \in \mathscr{D}$ and $t \in \mathscr{T}$ ). The usual vector notation is adopted.

Evidently, if $\varepsilon \neq 0$ then $\mathbf{u}$ and $\theta$ are coupled together. Further, if $\beta>0$, the system (1.1) is of (full) hyperbolic type; otherwise it is of (mixed) hyperbolic-parabolic type. If $\varepsilon=0$, then $\mathbf{u}$ and $\theta$ become uncoupled; in this case, the first equation in the system is hyperbolic and the second one is parabolic or hyperbolic according as $\beta=0$ or $\beta>0$.

* Received April 10, 1986. 
Systems of the type (1.1) arise in thermoelasticity. In fact, if $\mathbf{u}$ is regarded as a dimensionless displacement and $\theta$ as a dimensionless temperature and if appropriate physical meanings are attached to the constants $c, \alpha, \beta, \gamma$, and $\varepsilon$, the system (1.1) represents the field equations (in dimensionless form) of different models of the linear dynamical theory of homogeneous and isotropic thermoelasticity for different sets of values of the constants $\alpha, \beta$, and $\gamma$; actually, the case

$$
\text { (i) } \alpha \geqslant \beta>0, \gamma=0
$$

corresponds to the Green-Lindsay model $[1,2,3]$, the case

$$
\text { (ii) } \alpha=0, \beta=\gamma>0
$$

corresponds to the Lord-Shulman model $[4,5]$ and the case

$$
\text { (iii) } \alpha=\beta=\gamma=0
$$

corresponds to the conventional model $[6,7,8]$ of the theory. The field equations of all these three models of thermoelasticity may therefore be studied, in a unified way, by studying the system (1.1).

It may be pertinent to mention that in the Green-Lindsay and Lord-Shulman models, the system (1.1) is fully hyperbolic and (consequently) predicts a finite spread of propagation for thermoelastic disturbance, admitting the so-called "second sound" phenomenon. In the conventional model the system is of hyperbolic-parabolic type and consequently predicts an infinite spread of propagation, which is not realistic from a strict physical point of view. For a review of the relevant literature, see [9].

The object of this paper is to present three general, complete solutions of the coupled system of Eqs. (1.1). Each of these solutions is obtained in terms of a vector function and a scalar function obeying an uncoupled system of partial differential equations. In the first solution (presented in Sec. 2) the vector function obeys a second-order equation while the scalar function obeys a fourth-order equation. The same is the case with the second solution (presented in Sec. 3). In the last solution (presented in Sec. 4) the vector function obeys a sixth-order equation while the scalar function obeys a fourth-order equation. These solutions are, respectively, analogous to the Lamé, Papkovitch, and Galerkin solutions in classical elasticity $[10,11]$. The corresponding solutions valid in the three models of thermoelasticity, as determined by inequalities (1.3)-(1.5), emerge as particular cases. The connection among the solutions is also exhibited (in Sec. 5). The Lamé-type solution has also been used to uncouple the system (1.1) into a sixth-order equation for $\mathbf{u}$ and a fourth-order equation for $\theta$.

For convenience, we rewrite the system (1.1) as follows:

$$
\begin{gathered}
L_{1}(\mathbf{u}, \theta)+\mathbf{F}=\mathbf{0}, \\
L_{2}(\theta, \mathbf{u})+\left(1+\gamma \frac{\partial}{\partial t}\right) h=0 .
\end{gathered}
$$


Here

with

$$
\begin{aligned}
& L_{1}(\mathbf{u}, \theta)=D_{2} \mathbf{u}+a^{2} \nabla \operatorname{div} \mathbf{u}-\left(1+\alpha \frac{\partial}{\partial t}\right) \nabla \theta, \\
& L_{2}(\theta, \mathbf{u})=D_{3} \theta-\varepsilon \frac{\partial}{\partial t}\left(1+\gamma \frac{\partial}{\partial t}\right) \operatorname{div} \mathbf{u},
\end{aligned}
$$

$$
\begin{aligned}
& D_{2}=c^{2} \nabla^{2}-\frac{\partial^{2}}{\partial t^{2}}, \\
& D_{3}=\nabla^{2}-\frac{\partial}{\partial t}-\beta \frac{\partial^{2}}{\partial t^{2}},
\end{aligned}
$$

and

$$
a^{2}=1-c^{2}
$$

We also require the following operators:

$$
\begin{aligned}
& D_{1}=\nabla^{2}-\frac{\partial^{2}}{\partial t^{2}} \\
& D_{4}=a^{2} D_{3}-\varepsilon \frac{\partial}{\partial t}\left(1+\alpha \frac{\partial}{\partial t}\right)\left(1+\gamma \frac{\partial}{\partial t}\right), \\
& D_{5}=D_{3} D_{1}-\varepsilon \nabla^{2} \frac{\partial}{\partial t}\left(1+\alpha \frac{\partial}{\partial t}\right)\left(1+\gamma \frac{\partial}{\partial t}\right) .
\end{aligned}
$$

It may be verified that the following identities hold:

$$
\begin{aligned}
D_{1}-D_{2} & =a^{2} \nabla^{2}, \\
D_{5}-D_{4} \nabla^{2} & =D_{2} D_{3}, \\
a^{2} D_{5}-D_{1} D_{4} & =\varepsilon D_{2} \frac{\partial}{\partial t}\left(1+\alpha \frac{\partial}{\partial t}\right)\left(1+\gamma \frac{\partial}{\partial t}\right) .
\end{aligned}
$$

Throughout our analysis it is assumed that all functions entering the discussion are continuous/differentiable in $\mathscr{D} \times \mathscr{T}$ up to the required order and that the differential operators are commutative.

2. Lamé-type solution. Suppose $\phi$ is an arbitrary scalar field and $\psi$ is an arbitrary vector field satisfying the equațions

$$
\begin{gathered}
D_{5} \phi=D_{3} f-\left(1+\gamma \frac{\partial}{\partial t}\right) h, \\
D_{2} \psi=\mathbf{g},
\end{gathered}
$$

and put

$$
\begin{gathered}
\mathbf{u}=\left(1+\alpha \frac{\partial}{\partial t}\right)(\nabla \phi+\operatorname{curl} \psi), \\
\theta=D_{1} \phi-f .
\end{gathered}
$$

Here $f$ and $\mathbf{g}$ are defined by

$$
\mathbf{F}=-\left(1+\alpha \frac{\partial}{\partial t}\right)(\nabla f+\operatorname{curl} \mathbf{g})
$$


(Note that this type of representation of $\mathbf{F}$ is admissible in view of the Helmholtz resolution of a vector field.)

Substituting the expressions (2.3) into the right-hand sides of (1.7) and (1.8) and using (1.11) we obtain

$$
\begin{aligned}
& L_{1}(\mathbf{u}, \theta)=\left(1+\alpha \frac{\partial}{\partial t}\right)\left[D_{2}(\operatorname{curl} \psi)+\nabla f\right], \\
& L_{2}(\theta, \mathbf{u})=D_{5} \phi-D_{3} f .
\end{aligned}
$$

In view of Eqs. (2.1), (2.2), and (2.4), the system of Eqs. (1.6) is readily satisfied.

Thus, if $\phi$ and $\psi$ are arbitrary fields obeying Eqs. (2.1) and (2.2), then the system (2.3) is a solution of Eqs. (1.1). It may be noted that the functions $\phi$ and $\psi$ appearing in this solution are analogous to the Lamé potentials in classical elasticity [11].

We now show that this general solution is complete as well.

Suppose $\{\mathbf{u}, \theta\}$ is an arbitrary solution of the system (1.1). Then we have

$$
\begin{gathered}
D_{2} \mathbf{u}+a^{2} \nabla \operatorname{div} \mathbf{u}+\mathbf{F}_{0}=\mathbf{0}, \\
D_{3} \theta-\left(1+\gamma \frac{\partial}{\partial t}\right)\left[\varepsilon \frac{\partial}{\partial t} \operatorname{div} \mathbf{u}-h\right]=0,
\end{gathered}
$$

where

$$
\begin{aligned}
\mathbf{F}_{0} & =-\left(1+\alpha \frac{\partial}{\partial t}\right) \nabla \theta+\mathbf{F} \\
& =-\left(1+\alpha \frac{\partial}{\partial t}\right)[\nabla(f+\theta)+\operatorname{curl} \mathbf{g}]
\end{aligned}
$$

on using (2.4). Equation (2.7) is analogous to the Navier's equation of classical elastodynamics [11, p. 232]. Following the method employed in [11, p. 234] it is straightforward to show that $\mathbf{u}$, which obeys (2.7), has a representation of the form

$$
\mathbf{u}=\left(1+\alpha \frac{\partial}{\partial t}\right)(\nabla \phi+\operatorname{curl} \psi)
$$

where $\phi$ and $\psi$ meet the equations

$$
\begin{aligned}
& D_{1} \phi=f+\theta, \\
& D_{2} \psi=\text { g. }
\end{aligned}
$$

Substituting for $\mathbf{u}$ and $\theta$ from Eqs. (2.10) and (2.11) in Eq. (2.8) and using the identities (1.11), we find that $\phi$ is governed by the equation

$$
D_{5} \phi=D_{3} f-\left(1+\gamma \frac{\partial}{\partial t}\right) h .
$$

Equations (2.13) and (2.12) are identical with Eqs. (2.1) and (2.2). Thus every solution $\{\mathbf{u}, \theta\}$ of the system of Eqs. (1.1) admits a representation of the form (2.3), where $\phi$ and $\psi$ obey Eqs. (2.1) and (2.2). In other words, the solution described by Eqs. (2.1)-(2.4) is complete.

It may be noted that in the context of conventional thermoelasticity, a solution of the type (2.3) has been obtained by Deresiewicz [12] and by Zorski [13]; the completeness of this solution is presented in [8]. 
Uncoupling of the system. The solution described by Eqs. (2.1)-(2.4) may be utilized to decouple completely the system of Eqs. (1.1); we proceed as follows.

Eliminating $\phi$ from Eqs. (2.1) and $(2.3)_{2}$ and using (2.4) and $(1.10)_{3}$, we obtain the following equation that contains $\theta$ as the only unknown function:

$$
D_{5} \theta+\left(1+\gamma \frac{\partial}{\partial t}\right)\left[\varepsilon \frac{\partial}{\partial t} \operatorname{div} \mathbf{F}+D_{1} h\right]=0 .
$$

Together with (2.4) and (2.10), Eq. (2.13) yields

$$
D_{5} \operatorname{div} \mathbf{u}+D_{3} \operatorname{div} \mathbf{F}+\left(1+\alpha \frac{\partial}{\partial t}\right)\left(1+\gamma \frac{\partial}{\partial t}\right) \nabla^{2} h=0 .
$$

From Eqs. (2.13)-(2.15), we note that in the absence of $\mathbf{F}$ and $h$, the functions $\phi, \theta$, and div u satisfy one and the same equation: $D_{5} F=0$.

With the aid of Eqs. (2.14) and (2.15) and expressions (1.10), Eq. (1.6) ${ }_{1}$ reduces to the following equation which contains $\mathbf{u}$ as the only unknown function:

$$
D_{5} D_{2} \mathbf{u}-\nabla\left[D_{4} \operatorname{div} \mathbf{F}-\left(1+\alpha \frac{\partial}{\partial t}\right)\left(1+\gamma \frac{\partial}{\partial t}\right)\left(D_{2} h\right)\right]+D_{5} \mathbf{F}=\mathbf{0} .
$$

The coupled system of equations (1.1) has thus been decoupled into two independent Eqs. (2.14) and (2.16). Note that while each of the equations in the coupled system (1.1) is of order two, in the uncoupled system (2.14), (2.16), the equation governing $\theta$ is of order four and the equation governing $\mathbf{u}$ is of order six. The counterparts of Eqs. (2.14) and (2.16) in conventional thermoelasticity are presented in [8].

3. Papkovitch-type solution. Suppose $\Omega$ is an arbitrary vector field and $\omega$ is an arbitrary scalar field satisfying the equations

$$
\begin{gathered}
\left(1+\alpha \frac{\partial}{\partial t}\right) D_{2} \Omega=-\mathbf{F} \\
D_{5}(\omega+\mathbf{r} \cdot \Omega)=-\left(1+\gamma \frac{\partial}{\partial t}\right) h-D_{4} \operatorname{div} \Omega
\end{gathered}
$$

and put

$$
\begin{aligned}
& \mathbf{u}=\left(1+\alpha \frac{\partial}{\partial t}\right)[\nabla(\omega+\mathbf{r} \cdot \Omega)+\Omega], \\
& \theta=a^{2} \operatorname{div} \Omega+D_{1}(\omega+\mathbf{r} \cdot \Omega) .
\end{aligned}
$$

Substituting expressions (3.3) into the right-hand sides of (1.7) and (1.8) and using the identities (1.11), we obtain

$$
\begin{aligned}
& L_{1}(\mathbf{u}, \theta)=\left(1+\alpha \frac{\partial}{\partial t}\right) D_{2} \Omega, \\
& L_{2}(\theta, \mathbf{u})=D_{5}(\omega+\mathbf{r} \cdot \Omega)+D_{4} \operatorname{div} \Omega .
\end{aligned}
$$

In view of Eqs. (3.1) and (3.2), the system of equations (1.6) is readily satisfied.

Thus, if $\Omega$ and $\omega$ are arbitrary fields obeying Eqs. (3.1) and (3.2) then the system (3.3) is a solution of the system of Eqs. (1.1). It may be noted that the functions $\Omega$ and $\omega$ appearing in this solution are analogous to the Papkovitch potentials in classical elasticity $[10,11]$. 
We now show that this general solution is complete as well.

Suppose $\{\mathbf{u}, \theta\}$ is an arbitrary solution of the system (1.1). In view of the Helmholtz representation of a vector field, there exist a scalar field $p$ and a vector field $\mathbf{q}$ such that

$$
\mathbf{u}=\nabla p+\operatorname{curl} \mathbf{q} .
$$

We introduce two fields $\Omega$ and $\omega$ through the equations

$$
\begin{gathered}
\left(1+\alpha \frac{\partial}{\partial t}\right) \Omega=\operatorname{curl} \mathbf{q}+\nabla \omega_{0}, \\
\left(1+\alpha \frac{\partial}{\partial t}\right) \omega=p-\omega_{0}-\left(1+\alpha \frac{\partial}{\partial t}\right)(\mathbf{r} \cdot \Omega),
\end{gathered}
$$

where $\omega_{0}$ is defined by

$$
\omega_{0}(P, t)=-\frac{1}{4 \pi c^{2}} \int_{\mathscr{D}} \frac{\left\{D_{1} p-\left(1+\alpha \frac{\partial}{\partial t}\right) \theta\right\}(Q, t-R / c)}{R} d v .
$$

Here $P$ and $Q$ are points of $\mathscr{D}$ and $R$ is the distance from $P$ to $Q$, and integration is carried out with respect to $Q$.

Using the theory of retarded potentials [14], it may be verified that $\omega_{0}$ satisfies the equation

$$
D_{2} \omega_{0}=D_{1} p-\left(1+\alpha \frac{\partial}{\partial t}\right) \theta .
$$

Substituting for $p$ and curl q from Eqs. (3.8) and (3.7) in (3.6) and using (1.11), we obtain

$$
\mathbf{u}=\left(1+\alpha \frac{\partial}{\partial t}\right)[\nabla(\omega+\mathbf{r} \cdot \Omega)+\Omega]
$$

Together with Eqs. (3.7) and (3.8), Eq. (3.10) yields

$$
\left(1+\alpha \frac{\partial}{\partial t}\right)\left[\theta-\left\{a^{2} \operatorname{div} \Omega+D_{1}(\omega+\mathbf{r} \cdot \Omega)\right\}\right]=0 .
$$

For $\alpha=0$, this equation gives

$$
\theta=a^{2} \operatorname{div} \Omega+D_{1}(\omega+\mathbf{r} \cdot \Omega) .
$$

For $\alpha \neq 0$, (3.12) implies (3.13) provided

$$
\left[\theta-\left\{a^{2} \operatorname{div} \Omega+D_{1}(\omega+\mathbf{r} \cdot \Omega)\right\}\right]_{t=0}=0 .
$$

Therefore if we assume that the initial condition (3.14) holds when $\alpha \neq 0$, then (3.12) implies (3.13) in all the relevant cases.

Equations (3.11) and (3.13) are identical with the system (3.3) and therefore yield expressions (3.4) and (3.5). Since $\{\mathbf{u}, \theta\}$ is a solution of the system (1.6), it follows that $\Omega$ and $\omega$ satisfy Eqs. (3.1) and (3.2).

Thus, every solution $\{\mathbf{u}, \theta\}$ of the system (1.6) admits a representation of the form (3.3), where $\Omega$ and $\omega$ obey Eqs. (3.1) and (3.2); in the case when $\alpha \neq 0$, the initial condition (3.14) is also required to hold. Thus, under the assumptions made, the solution of the system (1.1) as described by Eqs. (3.1)-(3.3) is complete. 
Solutions analogous to (3.3) do exist in the literature on conventional thermoelasticity. For example, Sternberg and McDowell [15] have obtained a solution of the type (3.3) in the steady-state case; see also [16]. Biot [6] has obtained a similar solution in the quasi-static case; Verruijt [17] has deduced the completeness of this solution.

4. Galerkin-type solution. Suppose $\mathbf{G}$ is an arbitrary vector field and $H$ is an arbitrary scalar field satisfying the equations

$$
\begin{gathered}
D_{2} D_{5} \mathbf{G}=-\mathbf{F}, \\
D_{5} H=-\left(1+\gamma \frac{\partial}{\partial t}\right) h,
\end{gathered}
$$

and put

$$
\begin{aligned}
& \mathbf{u}=\nabla\left[\left(1+\alpha \frac{\partial}{\partial t}\right) H-D_{4} \operatorname{div} \mathbf{G}\right]+D_{5} \mathbf{G}, \\
& \theta=D_{1} H+\varepsilon D_{2} \frac{\partial}{\partial t}\left(1+\gamma \frac{\partial}{\partial t}\right)(\operatorname{div} \mathbf{G}) .
\end{aligned}
$$

Substituting expressions (4.3) into right-hand sides of (1.7) and (1.8) and using the identity $(1.11)_{1}$, we find that

$$
\begin{aligned}
& L_{1}(\mathbf{u}, \theta)=D_{2} D_{5} \mathbf{G}, \\
& L_{2}(\theta, \mathbf{u})=D_{5} H .
\end{aligned}
$$

In view of Eqs. (4.1) and (4.2), the system of equations (1.6) is readily satisfied.

Thus, if $\mathbf{G}$ and $H$ are arbitrary fields governed by Eqs. (4.1) and (4.2), the system (4.3) is a solution of the system of equations (1.1). It may be noted that the function $\mathbf{G}$ appearing in this solution is analogous to the Galerkin vector in classical elasticity $[10,11]$.

We now show that this general solution is complete as well.

Suppose $\{\mathbf{u}, \theta\}$ is an arbitrary solution of the system (1.1). In view of the Helmholtz representation, there exist a scalar field $p$ and a vector field $\mathbf{q}$ such that Eq. (3.6) holds.

We introduce the fields $\mathbf{G}$ and $H$ through the equations

$$
\begin{gathered}
\mathbf{G}=\nabla p_{0}+\operatorname{curl} \mathbf{q}_{0}, \\
\left(1+\alpha \frac{\partial}{\partial t}\right) H=p-D_{2} D_{3} p_{0},
\end{gathered}
$$

where $p_{0}$ and $\mathbf{q}_{0}$ are such that

$$
\begin{aligned}
D_{5} D_{2} p_{0}= & D_{1} p-\left(1+\alpha \frac{\partial}{\partial t}\right) \theta, \\
& D_{5} \mathbf{q}_{0}=\mathbf{q} .
\end{aligned}
$$

We note that the existence of functions $p_{0}$ and $\mathbf{q}_{0}$ as solutions of Eqs. (4.8) and (4.9) is guaranteed by the Cauchy-Kowalewski theorem.

From Eqs. (4.6) and (4.9) we have

$$
\begin{gathered}
\operatorname{div} \mathbf{G}=\nabla^{2} p_{0}, \\
D_{5} \mathbf{G}=D_{5} \nabla p_{0}+\operatorname{curl} \mathbf{q} .
\end{gathered}
$$


Substituting for $p$ and curl $\mathbf{q}$ from Eqs. (4.7) and (4.11) in Eq. (3.6) and taking note of (4.10), (1.10), and (1.11), we obtain

$$
\mathbf{u}=\nabla\left[\left(1+\alpha \frac{\partial}{\partial t}\right) H-D_{4} \operatorname{div} \mathbf{G}\right]+D_{5} \mathbf{G} .
$$

Together with Eqs. (4.7) and (4.10) as well as (1.10) and (1.11), Eq. (4.8) gives

$$
\left(1+\alpha \frac{\partial}{\partial t}\right)\left[\theta-\left\{D_{1} H+\varepsilon D_{2} \frac{\partial}{\partial t}\left(1+\gamma \frac{\partial}{\partial t}\right)(\operatorname{div} \mathbf{G})\right\}\right]=0
$$

For $\alpha=0$, this equation gives

$$
\theta=D_{1} H+\varepsilon D_{2} \frac{\partial}{\partial t}\left(1+\gamma \frac{\partial}{\partial t}\right)(\operatorname{div} \mathbf{G})
$$

For $\alpha \neq 0,(4.13)$ implies (4.14) provided

$$
\left[\theta-\left\{D_{1} H+\varepsilon D_{2} \frac{\partial}{\partial t}\left(1+\gamma \frac{\partial}{\partial t}\right)(\operatorname{div} \mathbf{G})\right\}\right]_{t=0}=0 .
$$

Therefore, if we assume that the initial condition (4.15) holds when $\alpha \neq 0$, then (4.13) implies (4.14) in all relevant cases.

Equations (4.12) and (4.14) are identical with the system (4.3) and therefore yield Eqs. (4.4) and (4.5). Since $\{\mathbf{u}, \theta\}$ is a solution of the system (1.6), it follows that $\mathbf{G}$ and $H$ meet Eqs. (4.1) and (4.2).

Thus, every solution $\{\mathbf{u}, \theta\}$ of the system (1.6) admits a representation of the form (4.3), where $\mathbf{G}$ and $H$ obey Eqs. (4.1) and (4.2); in the case when $\alpha \neq 0$, the initial condition (4.15) is also required to hold. Thus, under the assumptions made, the solution of the system (1.1) as described by Eqs. (4.1)-(4.3) is complete.

In the context of conventional thermoelasticity, a solution of the type (4.3) is given in [18] (without completeness); references to earlier works on such solutions may be found in $[8,18]$.

5. Connection among the solutions. A connection among the solutions (2.3), (3.3), and (4.3) may be established easily if the functions $\phi, \psi, \omega, \Omega$ and $H, \mathbf{G}$ are related through the equations

$$
\begin{aligned}
& \Omega=\operatorname{curl} \psi+\nabla \phi_{0}, \\
& \omega=\phi-\phi_{0}-(\mathbf{r} \cdot \Omega),
\end{aligned}
$$

where $\phi_{0}$ is given by

$$
\phi_{0}(P, t)=-\frac{1}{4 \pi c^{2}} \int_{\mathscr{D}} \frac{f(Q, t-R / c)}{R} d v
$$

and

$$
\begin{gathered}
\left(1+\alpha \frac{\partial}{\partial t}\right) \Omega=D_{5} \mathbf{G} \\
\left(1+\alpha \frac{\partial}{\partial t}\right)(H-\omega-\mathbf{r} \cdot \Omega)=D_{4} \operatorname{div} \mathbf{G}
\end{gathered}
$$


It is straightforward to verify that if we substitute for $\phi$ and $\psi$ from relations (5.1) in Eqs. (2.1)-(2.3), taking note of Eqs. (1.11) and (2.4) and the fact that $D_{2} \phi_{0}=f$, we obtain Eqs. (3.1)-(3.3). Similarly, if we substitute for $\omega$ and $\Omega$ from relations (5.3), taking note of Eqs. (1.11) and assuming homogeneous initial conditions, we obtain Eqs. (4.1)-(4.3). Other interrelationships among the solutions also follow from relations (5.1)-(5.3).

6. Closing remarks. The solution (2.3) determines $\mathbf{u}$ and $\theta$ in terms of the functions $\phi$ and $\psi$ satisfying two uncoupled Eqs. (2.1) and (2.2) which are of order four and two, respectively. The solution (4.3) determines $\mathbf{u}$ and $\theta$ in terms of $\mathbf{G}$ and $H$ satisfying two uncoupled Eqs. (4.1) and (4.2) which are of order six and four, respectively. The solution (3.3) determines $\mathbf{u}$ and $\theta$ in terms of $\Omega$ and $\omega$ satisfying Eqs. (3.1) and (3.2) which are of order two and four, respectively. Note that, unlike the other two cases, equations governing $\Omega$ and $\omega$ are not completely uncoupled. In this case, Eq. (3.1) is to be solved first to determine $\Omega$ and then (after knowing $\Omega$ ) Eq. (3.2) is to be solved for $\omega$. Every (solvable) problem governed by the coupled system (1.1) may therefore be solved by employing Eqs. (2.1)-(2.2), (3.1)-(3.2), or (4.1)-(4.2).

In obtaining the solutions (2.3), (3.3), and (4.3), no restrictions have been imposed on the parameters $c, \alpha, \beta, \gamma$, and $\varepsilon$. As such, these solutions are valid for a broad class of problems governed by equations of the type (1.1). If restrictions (1.3), (1.4), or (1.5) are imposed, the solutions correspond to those valid in the Green-Lindsay model, the Lord-Shulman model, or the conventional model (respectively) of thermoelasticity. Counterparts of the uncoupled Eqs. (2.14) and (2.16) in the three models of thermoelasticity may also be deduced similarly. It may be mentioned that in the contexts of the Lord-Shulman and the Green-Lindsay models of thermoelasticity, solutions of the types (3.3) and (4.3) and equations of the types (2.14) and (2.16) have not been obtained earlier. Solution of the type (3.3) is not found in the literature on conventional dynamic thermoelasticity also.

In view of the "Sternberg criteria" [11, pp. 236, 237], it may be remarked that the Lamé-type solution is generally more useful than the other two solutions in applications. But, in the treatment of specific problems (such as those considered in $[15,18]$ ), the Papkovitch-type as well as the Galerkin-type solutions might exhibit certain advantages.

Acknowledgments. The author is thankful to the referee for his valuable comments and suggestions. This work was partially supported by a research grant (No. F. 8-3/84 SR III) received from the University Grants Commission, New Delhi.

\section{REFERENCES}

[1] A. E. Green and K. A. Lindsay, Thermoelasticity, J. Elasticity 2, 1-7 (1972)

[2] A. E. (jreen, A note on linear thermoelasticity, Mathematika 19, 69-75 (1972)

[3] D. S. Chandrasekharaiah, Wave propagation in a thermoelastic half-space, Indian J. Pure. Appl. Math. 12, 226-241 (1981)

[4] H. W. Lord and Y. Shulman, A generalized dynamical theory of thermoelasticity, J. Mech. Phys. Solids 15, 299-309 (1967)

[5] D. S. Chandrasekharaiah, On generalized thermoelastic wave propagation, Proc. Indian Acad. Sci. (Math. Sci). 89, 43-52 (1980)

[6] M. A. Biot, Thermoelasticity and irrecersible thermodynamics, J. Appl. Phys. 27, 240-253 (1956) 
[7] P. Chadwick, Thermoelasticity. The dynamic theory, Progress in Solid Mechanics (Ed. R. Hill and I. N. Sneddon) Vol. 1, North Holland, Amsterdam, 1960

[8] D. E. Carlson, Linear Thermoelasticity, Encyclopedia of Physics, Vol. VI a/2, Springer-Verlag, New York, 1972

[9] D. S. Chandrasekharaiah, Thermoelasticity with second sound-A review, Appl. Mech. Rev. 39, 355-376 (1986)

[10] I. S. Sokolnikoff, Mathematical theory of elasticity, 2nd Ed., McGraw-Hill, New York, 1956

[11] M. E. Gurtin, The linear theory of elasticity, Encyclopedia of Physics. Vol VI a/2, Springer-Verlag, New York, 1972

[12] H. Deresiewicz, Solution of the equations of thermoelasticity, Proc. 3rd U.S. National Congr. Appl. Mech., Brown University, 287-291 (1958)

[13] H. Zorski, Singular solutions for thermoelastic media, Bull. Acad. Poln. Sci. Techn. 6, 331-339 (1958)

[14] H. B. Phillips, Vector Analysis, John Wiley, New York, 1933

[15] E. Sternberg and E. L. McDowell, On the steady state thermoelastic problem for the half-space, Quart. Appl. Math. 14, 381-398 (1957)

[16] J. L. Nowinski, Theory of thermoelasticity with applications, p. 363, Sijthoff-Noordhoff, Alphen Aan Den Rijn, 1978

[17] A. Verruijt, The completeness of Biot's solution of the coupled thermoelastic problem, Quart. Appl. Math. 26. 485-490 (1969)

[18] W. Nowacki, Dynamic problems of Thermoelasticity, Noordhoff, Leyden, pp. 26-29, 1975 